\title{
The association of cardiovascular disease with impaired health-related quality of life among patients with type 2 diabetes mellitus
}

\author{
Mun Chieng $\operatorname{Tan}^{1}$, MSc, Ooi Chuan Ng², MBBS, MRCP, Teck Wee Wong ${ }^{3}$, MRCP, FAMS, Abdul Rahman Hejar ${ }^{4}$, MD, MPH,
} Joseph Anthony ${ }^{2}$, MBBS, M Int Med, Harri Sintonen ${ }^{5}$, PhD

\begin{abstract}
INTRODUCTION The aim of this study was to evaluate the health-related quality of life (HRQLL) of Malaysian patients with type 2 diabetes mellitus (T2DM) who have cardiovascular disease (CVD), as well as identify the determinants of HRQoL among this cohort of patients.

METHODS This study was an analytical cross-sectional study involving 313 patients aged 30-78 years (150 men, 163 women; mean age $55.7 \pm 9.2$ years) who were diagnosed with T2DM (mean duration of T2DM 10.1 \pm 8.1 years) at two tertiary Malaysian government hospitals. The patients' sociodemographic, lifestyle, clinical and laboratory data were collected prospectively from medical records and via face-to-face interviews. HRQoL was assessed using the 15D instrument - a generic, 15-dimensional and standardised measure of HRQoL that can be used as both a profile and a single index score measure.

RESULTS T2DM patients with CVD were found to have significantly lower 15D HRQoL scores than their nonCVD counterparts $(p<0.001)$. The HRQoL of T2DM patients with CVD was significantly lower than those without CVD $(p<0.05)$ in all of the 15 dimensions of the 15D instrument. Multinomial logistic regression analysis using backward stepwise method revealed a significant association between CVD and impaired HRQoL (odds ratio [OR] 11.746, 95\% confidence interval [CI] 4.898-28.167). Age (OR 1.095, 95\% Cl 1.054-1.137), duration of T2DM (OR 1.085, 95\% Cl 1.032-1.140), ethnicity (OR 0.411, 95\% Cl 0.187-0.903), body mass index (OR $1.074,95 \% \mathrm{Cl}$ 1.006-1.148), and physical activity level (OR 3.506, 95\% Cl 1.415-8.689) were also significant predictors of HRQoL. CONCLUSION In T2DM patients, the presence of CVD was significantly associated with a lower HRQoL. Therefore, the importance of tertiary prevention to minimise the potential deterioration of the HRQLL of T2DM patients with CVD should be highly emphasised.
\end{abstract}

Keywords: 15D instrument, cardiovascular disease, health-related quality of life, type 2 diabetes mellitus

\section{INTRODUCTION}

Diabetes mellitus (DM) is one of the fastest growing major public health problems worldwide. It is predicted that approximately 552 million adults will have DM by 2030.(1) There are three types of DM, of which type 2 DM (T2DM) is the most prevalent. Due to its prevalence, the cost of managing the disease results in considerable economic and financial burden to the society and healthcare sector. The disease also causes varying amounts of distress to its sufferers. Although there have been improvements in the quality of care of T2DM patients, chronic long-term complications remain an important and costly problem to both the individual and society. ${ }^{(2)}$ Epidemiological studies have indicated that patients with T2DM have a 2-4 times higher risk of cardiovascular disease (CVD) mortality than those without DM, ${ }^{(3-7)}$ with CVD being the principal cause of death in patients with T2DM..$^{(3,8-13)}$

CVD is associated with premature mortality and a reduced quality of life (QoL). CVD not only results in physical disability, heavy economic cost, lifelong medication, and psychological burden, it also leads to compromised health-related quality of life (HRQoL). ${ }^{(14)}$ Objective clinical measures of cardiovascular status, such as those that use the New York Heart Association classification, ${ }^{(15)}$ cholesterol values, blood pressure readings, electrocardiography (ECG) findings, and physiologic functions (e.g. left ventricular ejection fraction), are of interest mainly due to their known associations with poor health outcomes. Although these measures are objective, they are unable to provide accurate information on the subjective QoL of human beings. The objective of healthcare includes both prolonging life and enhancing QoL. The aim of medical interventions is not only to extend life, but also to relieve symptoms and improve the patient's function and ability to participate in daily life activities. ${ }^{(16)}$ Consequently, HRQoL measures have gained increasing attention as outcome variables in studies evaluating modern treatments of CVD, especially studies on coronary artery disease (CAD). ${ }^{(16)}$

Although HRQoL has been increasingly applied as an indicator of health outcome in recent years, and HRQoL reflects 'total welfare' through its capture of all relevant

\footnotetext{
${ }^{1}$ Department of Nutrition and Dietetics, ${ }^{2}$ Department of Medicine, Faculty of Medicine and Health Sciences, Universiti Putra Malaysia, Selangor, ${ }^{3}$ Heart and Lung Centre, iHEAL Medical Centre Kuala Lumpur, Kuala Lumpur, ${ }^{4}$ Department of Community Health, Faculty of Medicine and Health Sciences, Universiti Putra Malaysia, Selangor, Malaysia, ${ }^{5}$ Department of Public Health, Hjelt Institute, University of Helsinki, Finland

Correspondence: Ms Mun Chieng Tan, Graduate Research Fellow, Department of Nutrition and Dietetics, Faculty of Medicine and Health Sciences, Universiti Putra Malaysia, 43400 UPM Serdang, Selangor Darul Ehsan, Malaysia.mun_chieng_tan@yahoo.com
} 
dimensions of health of an individual, little is known about the relationship between CVD and HRQoL in T2DM patients. We thus conducted the present study to compare the HRQoL of T2DM patients with and without CVD. The possible differences in the quality and quantity of suffering resulting from the patient's illness were examined and evaluated using the general, non-disease-specific 15D instrument. ${ }^{(17)}$ As the present study is an original research, we believe that it will play an important role in the improvement of T2DM patient care and provide evidence on the need for cardiac rehabilitation programmes in Malaysia. The data obtained in the present study can also be used to identify the features of the disease and the treatment strategies that future research should concentrate on in the effort to improve the well-being of T2DM patients in a more cost-effective manner.

\section{METHODS}

The present cross-sectional study comprised a systematic random sample of 313 T2DM patients from two tertiary hospitals in Klang Valley, Malaysia (one in the federal territory of Kuala Lumpur, and the other in Selangor). The study cohort contained patients from the three main ethnic groups in Malaysia - Malay, Chinese and Indian. T2DM patients were identified via the hospitals' medical records. Identified patients were recruited by trained, multilingual interviewers during the patients' routine visits to the hospitals between June 2010 and April 2011. Patients who were ambulatory and diagnosed with T2DM were included in the present study. The following patients were excluded: (a) patients with type $1 \mathrm{DM}$ or gestational DM; (b) patients who were pregnant or lactating; (c) patients with the following diseases/conditions: nonatherosclerotic heart diseases (e.g. rheumatic heart disease and congestive heart failure secondary to thyrotoxicosis), atrial fibrillation, venous thromboembolism, nonischaemic cardiomyopathy, valvular heart disease, cardiac dysrhythmias, inflammatory heart disease (e.g. endocarditis, pericarditis and myocarditis), congenital heart disease, embolic and haemorrhagic strokes, malignant disease, severe psychiatric illness and dementia; and (d) patients with a prosthetic heart valve.

Prior to the interview session, written informed consent was obtained from all patients who agreed to participate in the present study. All participating patients were informed about the nature of the study, and participation was completely voluntary. The study's protocol was reviewed and approved by both the Medical Research Ethical Committee of the Faculty of Medicine and Health Sciences, Universiti Putra Malaysia, Malaysia, and the Ministry of Health, Malaysia.

Sociodemographic and lifestyle data including age, gender, level of education, marital status, employment status, dietary intake (using the 24-hr dietary recall method), ${ }^{(18)}$ level of physical activity (using the International Physical Activity Questionnaire), (19) smoking status and alcohol consumption behaviour were collected. The following clinical and laboratory characteristics were recorded: CVD-related characteristics (described in the following paragraph), current medication, duration of T2DM, glycaemic control (fasting plasma glucose and $\mathrm{HbA} 1 \mathrm{c}$ levels), systolic and diastolic blood pressure, body mass index (BMI) and waist-to-hip ratio (WHR).

In the present study, CVD was defined by the presence of one or more of the following: CAD (e.g. nonfatal myocardial infarction, angina, and a history of coronary artery bypass graft or angioplasty), nonfatal ischaemic stroke (silent brain infarction and haemorrhagic stroke were excluded), transient ischaemic attack, and clinically significant peripheral vascular disease (PVD). The presence of CVD was established based on physical examination and the patient's medical history. For each positive response given during the face-to-face interview, interviewers inquired about the current status of the condition and the use of medications for the condition. Participants who responded affirmatively to any of the CVD questions had their extracted medical records reviewed by our research team's adjudication committee, which consisted of a consultant interventional cardiologist, consultant endocrinologists and physicians.

HRQoL was measured using 15D, a generic, comprehensive, multidimensional, standardised, self-administered instrument, which has both a profile and single index score property. ${ }^{(17)}$ Conceptually, the 15D instrument subscribes to the World Health Organization's definition of health (i.e. health is composed of physical, mental and social well-being). ${ }^{(20)}$ The 15D instrument describes health status in 15 dimensions mobility, vision, hearing, breathing, sleeping, eating, speech, elimination (i.e. excretory functions), usual activities, mental function, discomfort and symptoms, depression, distress, vitality, and sexual activity. Each dimension is divided into five ordinal response levels, ranked from the best possible health condition (i.e. score 1) to the worst (i.e. score 5). ${ }^{(17)}$ The patients were instructed to choose the response that best described their state of health at the moment. The valuation system of the 15D instrument is based on an application of the multiattribute utility theory. A set of preference weights (elicited from representative samples of the general population through a three-stage valuation procedure with a combined rating scale and magnitude estimation method) was used to generate level values for each dimension on a $0-1$ scale. The preference weights were then aggregated additively into an overall HRQoL $15 \mathrm{D}$ score (single index number) over all the dimensions. The maximum score is 1 (i.e. no problems on any dimension) and the minimum score is 0 (i.e. dead). ${ }^{(17)}$

The minimal clinically important difference in the overall score of the $15 \mathrm{D}$ instrument was set at 0.03 . In other words, a change of approximately \pm 0.03 in the score was regarded as important because, on average, such a change in the score indicates a tangible difference. ${ }^{(21)}$ As national scoring weights for this instrument were not available, the original Finnish scoring was used in the present study. Based on repeat measurements, 
the $15 \mathrm{D}$ instrument has been shown to be highly reliable, sensitive and responsive to change. ${ }^{(17,21-23)}$ To minimise missing data and inter-examiner error in the present study, all interviews were conducted by the same group of interviewers, all of whom were trained for the purposes of the study. The study cohort was divided into tertiles and the associations of CVD with other selected variables were analysed (highest 15D tertile vs the middle and lowest 15D tertiles). In the present study, the Cronbach's alpha reliability coefficient of the $15 \mathrm{D}$ instrument was 0.810 .

All statistical analyses were performed using IBM SPSS Statistics software version 21.0 (IBM Corp, Armonk, NY, USA). A p-value of $<0.05$ was considered statistically significant. Sociodemographic characteristics and risk factors were expressed as frequencies and percentages for categorical variables, and as mean \pm standard deviation (SD) for continuous variables. Group comparisons for normally distributed data were done using independent $t$-test, while nonparametric Mann-Whitney $U$ test was used for skewed data. Chi-square test was used to compare the differences in the categorical variables between the groups. Mann-Whitney $U$ test was performed, as the total scores and the scores of all the 15D dimensions of the study participants with and without CVD were not normally distributed. Using a stepwise backward multinomial logistic regression analysis of the highest 15D tertile versus the middle and lowest 15D tertiles, we studied associations between HRQoL and CVD status (absent vs present), adjusted for the following 11 background variables: age, duration of T2DM, BMI, WHR, gender, ethnicity, marital status, education level, smoking status, alcohol consumption, and physical activity. Covariates with the highest $p$-value were excluded sequentially. In this way, stepwise backward multinomial logistic regression analysis was continued until the most parsimonious model that still explained the data was found at $p$-value $<0.05$.

\section{RESULTS}

Among the 313 T2DM patients in our cohort, 113 (36.1\%) had CVD and 200 (63.9\%) did not. Table I shows the prevalence of CVD and its components in our study cohort. The T2DM patients with CVD experienced one or more cardiovascular events. The prevalence of CAD, cerebrovascular disease, and PVD were found to be $30.7 \%, 10.2 \%$ and $5.1 \%$, respectively.

Neuropathy $(41.5 \%)$ was found to be the main microvascular complication among the T2DM patients, followed by nephropathy $(17.6 \%)$ and retinopathy $(15.0 \%)$. The severity of nephropathy in the patients ranged from mild renal impairment to end-stage renal failure; only two patients required haemodialysis. Among the 313 T2DM patients, $18.8 \%$ patients received treatment with cardiac drugs, including glyceryl trinitrate $(5.8 \%)$, isosorbide mononitrate $(1.9 \%)$, isosorbide dinitrate $(6.7 \%)$, trimetazidine $(7.7 \%)$, digoxin $(1.3 \%)$, spironolactone (1.6\%), and frusemide (10.2\%) (Table II). A large
Table I. Cardiovascular disease-related characteristics of the study cohort $(n=313)$.

\begin{tabular}{lc}
\hline Variable & No. (\%) \\
\hline Cardiovascular disease & $113(36.1)$ \\
Coronary artery disease & $96(30.7)$ \\
Myocardial infarction & $34(10.9)$ \\
Angina pectoris & $69(22.0)$ \\
CABG & $14(4.5)$ \\
PTCA & $29(9.3)$ \\
Cerebrovascular disease & $32(10.2)$ \\
Ischaemic stroke & $30(9.6)$ \\
Transient ischaemic attack & $14(4.5)$ \\
Peripheral vascular disease & $16(5.1)$ \\
Diabetic foot ulcer & $9(2.9)$ \\
Gangrene/amputation & $0(0)$ \\
\hline
\end{tabular}

CABG: coronary artery bypass grafting; PTCA: percutaneous transluminal coronary angioplasty

Table II. Cardiovascular drug use of the study cohort $(n=313)$.

\begin{tabular}{|c|c|}
\hline Drug & No. (\%) \\
\hline Cardiac drugs* & $59(18.8)$ \\
\hline Antihypertensive drugs & $251(80.2)$ \\
\hline $\mathrm{CCBs}$ & $155(49.5)$ \\
\hline ACE inhibitors & $137(43.8)$ \\
\hline Beta blockers & $109(34.8)$ \\
\hline ARBs & $75(24.0)$ \\
\hline Diuretics & $36(11.5)$ \\
\hline Others $^{\dagger}$ & $23(7.3)$ \\
\hline Lipid-lowering agents & $279(89.1)$ \\
\hline Statins & $271(86.6)$ \\
\hline Fibrates & $24(7.7)$ \\
\hline Antiplatelets ${ }^{\ddagger} /$ anticoagulants $^{\S}$ & $165(52.7)$ \\
\hline
\end{tabular}

* Includes glyceryl trinitrate, isosorbide mononitrate, isosorbide dinitrate, trimetazidine, digoxin, spironolactone and frusemide. ${ }^{\dagger}$ Includes prazosin and doxazosin. FIncludes aspirin, ticlopidine, clopidogrel and dipyridamole. ${ }^{\S}$ Includes warfarin. ACE: angiotensin-converting enzyme; ARBs: angiotensin II receptor blockers; CCB: calcium channel blockers

percentage of the patients were on antihypertensive drugs (80.2\%); a larger proportion of patients with CVD (91.2\%) used antihypertensive drugs as compared to the patients without CVD $(74.0 \%)(p<0.001)$. Beta blockers were also used by a greater number of patients with CVD (61.1\%) than those without CVD (20.0\%) ( $p<0.001)$. Lipid-lowering drugs were used by $89.1 \%$ of the patients. Patients with CVD were also on more aggressive treatment for dyslipidaemia as compared to patients without CVD (93.8\% vs $86.5 \%$ ) ( $p=0.046)$. There was also a higher percentage of patients with CVD on statins than those without $(92.9 \%$ vs $83.0 \%)$ ( $p=0.013)$. The use of antiplatelets/anticoagulants, including aspirin, ticlopidine, clopidogrel, dipyridamole and warfarin, was reported in $52.7 \%$ patients.

The sociodemographic, clinical, and laboratory characteristics of the study cohort are presented in Table III. The mean age of all of the patients was $55.7 \pm 9.2$ years (range 30-78 years) and $52.1 \%$ were women. In terms of ethnicity, approximately half of the patients were of Malay ethnicity, followed by patients of Indian and Chinese ethnicities. The majority of our study cohort had a low educational status (i.e. a secondary school education or lower). The majority of the 
Table III. Sociodemographic and clinical characteristics of the study cohort $(n=313)$.

\begin{tabular}{|c|c|c|c|c|}
\hline \multirow[t]{2}{*}{ Characteristic } & \multicolumn{3}{|c|}{ No. (\%) } & \multirow[t]{2}{*}{ p-value ${ }^{+}$} \\
\hline & $\begin{array}{c}\text { All } \\
(n=313)\end{array}$ & $\begin{array}{l}\text { CVD absent } \\
(\mathrm{n}=\mathbf{2 0 0})\end{array}$ & $\begin{array}{l}\text { CVD present } \\
(n=113)\end{array}$ & \\
\hline Age* (yrs) $^{*}$ & $55.7 \pm 9.2$ & $54.7 \pm 9.7$ & $57.4 \pm 8.1$ & $0.013^{*}$ \\
\hline Known duration of T2DM* (yrs) & $10.1 \pm 8.1$ & $9.2 \pm 7.6$ & $11.5 \pm 8.7$ & $0.021^{*}$ \\
\hline $\begin{array}{l}\text { Gender } \\
\text { Male } \\
\text { Female }\end{array}$ & $\begin{array}{l}150(47.9) \\
163(52.1)\end{array}$ & $\begin{array}{r}84(42.0) \\
116(58.0)\end{array}$ & $\begin{array}{l}66(58.4) \\
47(41.6)\end{array}$ & $0.005^{*}$ \\
\hline $\begin{array}{l}\text { Ethnicity } \\
\text { Malay } \\
\text { Chinese } \\
\text { Indian }\end{array}$ & $\begin{array}{r}147(47.0) \\
80(25.6) \\
86(27.5)\end{array}$ & $\begin{array}{r}106(53.0) \\
47(23.5) \\
47(23.5)\end{array}$ & $\begin{array}{l}41(36.3) \\
33(29.2) \\
39(34.5)\end{array}$ & $0.015^{\ddagger}$ \\
\hline $\begin{array}{l}\text { Education level } \\
\text { No formal education } \\
\text { Primary school } \\
\text { Secondary school } \\
\text { College/university }\end{array}$ & $\begin{aligned} & 30(9.6) \\
& 72(23.0) \\
& 138(44.1) \\
& 73(23.3)\end{aligned}$ & $\begin{array}{l}20(10.0) \\
41(20.5) \\
83(41.5) \\
56(28.0)\end{array}$ & $\begin{array}{l}10(8.8) \\
31(27.4) \\
55(48.7) \\
17(15.0)\end{array}$ & 0.054 \\
\hline $\begin{array}{l}\text { Marital status } \\
\text { Married } \\
\text { Single/widowed/divorced }\end{array}$ & $\begin{array}{r}254(81.2) \\
59(18.8)\end{array}$ & $\begin{array}{r}165(82.5) \\
35(17.5)\end{array}$ & $\begin{array}{l}89(78.8) \\
24(21.2)\end{array}$ & 0.417 \\
\hline $\begin{array}{l}\text { Employment } \\
\text { Employed } \\
\text { Unemployed/retired } \\
\text { Housewives }\end{array}$ & $\begin{array}{r}134(42.8) \\
105(33.5) \\
74(23.6)\end{array}$ & $\begin{array}{l}86(43.0) \\
61(30.5) \\
53(26.5)\end{array}$ & $\begin{array}{l}48(42.5) \\
44(38.9) \\
21(18.6)\end{array}$ & 0.178 \\
\hline Total energy intake* (kcal/day) & $1674 \pm 694$ & $1579 \pm 590$ & $1843 \pm 824$ & $0.001^{*}$ \\
\hline $\begin{array}{l}\text { PA level } \\
\text { Low PA } \\
\text { Moderate PA } \\
\text { High PA }\end{array}$ & $\begin{array}{r}72(23.0) \\
71(22.7) \\
170(54.3)\end{array}$ & $\begin{array}{r}34(17.0) \\
35(17.5) \\
131(65.5)\end{array}$ & $\begin{array}{l}38(33.6) \\
36(31.9) \\
39(34.5)\end{array}$ & $<0.001^{*}$ \\
\hline $\begin{array}{l}\text { Smoking status } \\
\text { Current } \\
\text { Former } \\
\text { Never }\end{array}$ & $\begin{aligned} & 31(9.9) \\
64 & (20.4) \\
218 & (69.6)\end{aligned}$ & $\begin{array}{r}14(7.0) \\
34(17.0) \\
152(76.0)\end{array}$ & $\begin{array}{l}17(15.0) \\
30(26.5) \\
66(58.4)\end{array}$ & $0.004^{\ddagger}$ \\
\hline $\begin{array}{l}\text { Alcohol consumption } \\
\text { Current } \\
\text { Former } \\
\text { Never }\end{array}$ & $\begin{array}{c}16(5.1) \\
17(5.4) \\
280(89.5)\end{array}$ & $\begin{aligned} 7 & (3.5) \\
7 & (3.5) \\
186 & (93.0)\end{aligned}$ & $\begin{array}{c}9(8.0) \\
10(8.8) \\
94(83.2)\end{array}$ & $0.025^{\ddagger}$ \\
\hline$B M I^{*}\left(\mathrm{~kg} / \mathrm{m}^{2}\right)$ & $29.0 \pm 5.0$ & $29.2 \pm 5.4$ & $28.5 \pm 4.2$ & 0.259 \\
\hline WHR $^{*}$ & $0.9 \pm 0.1$ & $0.9 \pm 0.1$ & $1.0 \pm 0.1$ & $0.002^{\ddagger}$ \\
\hline $\begin{array}{l}\text { T2DM treatment } \\
\text { Only oral agents } \\
\text { Only insulin } \\
\text { Oral agents and insulin }\end{array}$ & $\begin{array}{l}190(60.7) \\
25(8.0) \\
98(31.3)\end{array}$ & $\begin{aligned} 120 & (60.0) \\
17 & (8.5) \\
63 & (31.5)\end{aligned}$ & $\begin{array}{c}70(61.9) \\
8(7.1) \\
35(31.0)\end{array}$ & 0.890 \\
\hline Receiving treatment for hypertension & $251(80.2)$ & $148(74.0)$ & $103(91.2)$ & $<0.001^{*}$ \\
\hline Receiving treatment for dyslipidaemia & $279(89.1)$ & $173(86.5)$ & $106(93.8)$ & $0.046^{*}$ \\
\hline $\begin{array}{l}\text { HbA1c level } \\
\leq 7.0 \% \text { (good) } \\
>7.0 \% \text { (poor) }\end{array}$ & $\begin{array}{r}69(22.0) \\
244(78.0)\end{array}$ & $\begin{array}{r}43(21.5) \\
157(78.5)\end{array}$ & $\begin{array}{l}26(23.0) \\
87(77.0)\end{array}$ & 0.757 \\
\hline FPG* (mmol/L) & $8.8 \pm 3.6$ & $8.7 \pm 3.5$ & $9.0 \pm 3.9$ & 0.562 \\
\hline $\mathrm{SBP}^{*}(\mathrm{mmHg})$ & $137.9 \pm 18.9$ & $138.8 \pm 17.7$ & $136.5 \pm 20.9$ & 0.302 \\
\hline $\mathrm{DBP}^{*}(\mathrm{mmHg})$ & $80.7 \pm 11.8$ & $81.4 \pm 11.4$ & $79.4 \pm 12.6$ & 0.154 \\
\hline Total cholesterol* (mmol/L) & $4.9 \pm 1.3$ & $4.9 \pm 1.1$ & $5.0 \pm 1.6$ & 0.527 \\
\hline LDL-C* $(\mathrm{mmol} / \mathrm{L})$ & $2.9 \pm 1.1$ & $2.9 \pm 0.9$ & $3.0 \pm 1.4$ & 0.465 \\
\hline HDL-C* (mmol/L) & $1.2 \pm 0.3$ & $1.2 \pm 0.4$ & $1.1 \pm 0.3$ & $<0.001^{*}$ \\
\hline Triglycerides* $(\mathrm{mmol} / \mathrm{L})$ & $1.8 \pm 1.2$ & $1.7 \pm 1.2$ & $2.1 \pm 1.3$ & $0.013^{*}$ \\
\hline Overall 15D score* & $0.8713 \pm 0.1079$ & $0.9132 \pm 0.0788$ & $0.7972 \pm 0.1128$ & $<0.001^{\neq, \S}$ \\
\hline
\end{tabular}

${ }^{*}$ Data is presented as mean \pm standard deviation. ${ }^{+}$Independent $t$-test and chi-square test used unless indicated. ${ }^{*}$ Statistically significant (i.e. $p<0.05$ ). $\S$ Mann-Whitney $U$ test used.

15D: 15-dimension instrument to access health-related quality of life; BMI: body mass index; CVD: cardiovascular disease; DBP: diastolic blood pressure; FPG: Fasting plasma glucose; HbA1c: glycosylated haemoglobin; HDL-C: high-density lipoprotein cholesterol; LDL-C: low-density lipoprotein cholesterol; PA: physical activity; SBP: systolic blood pressure; T2DM: type 2 diabetes mellitus; WHR: waist-to-hip ratio 


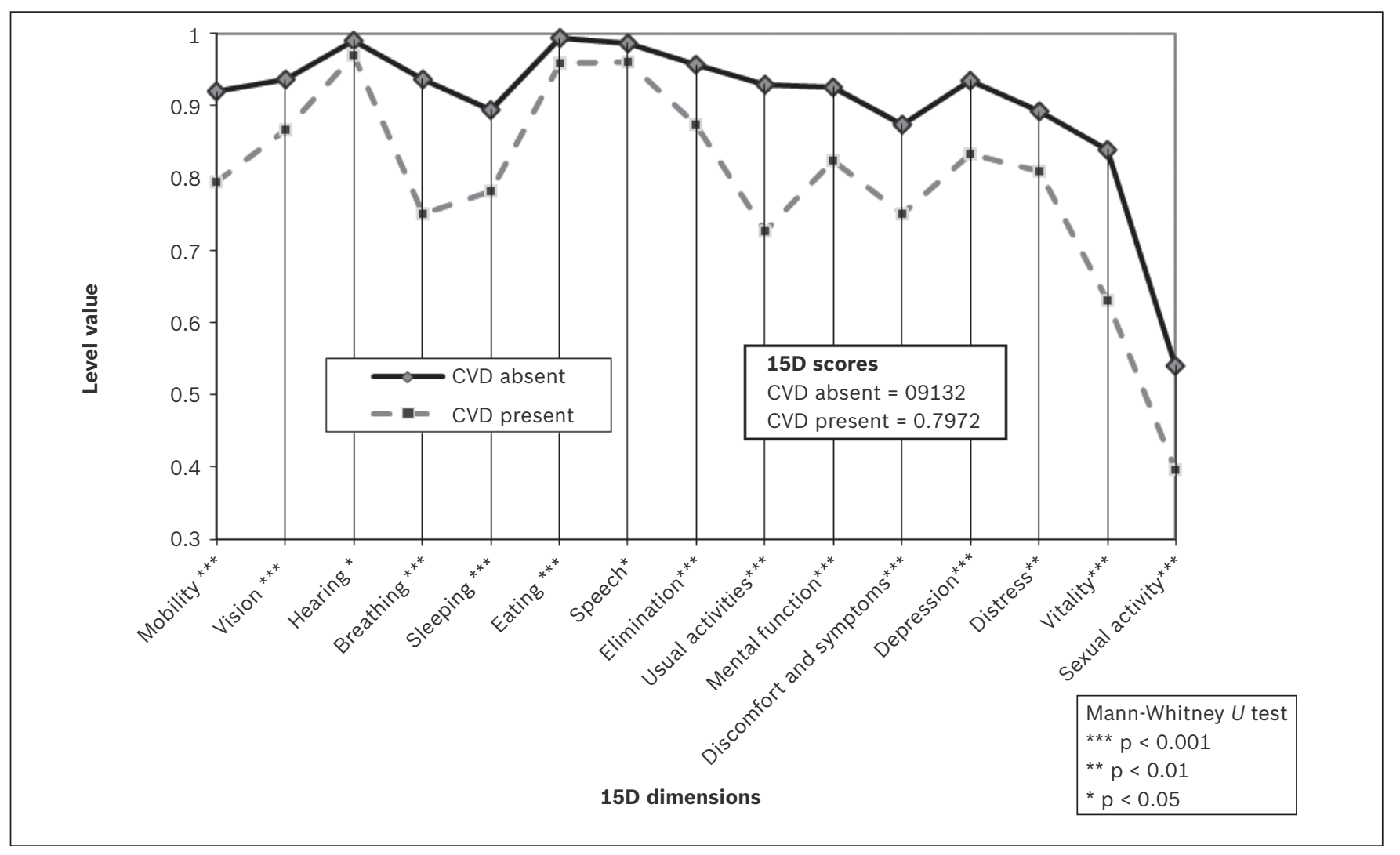

Fig. 1 The 15D profile of the study cohort, according to their cardiovascular disease status.

Table IV. The prevalences of cardiovascular disease and its components in the study cohort, according to the 15D tertiles.

\begin{tabular}{|c|c|c|c|c|}
\hline \multirow[t]{2}{*}{ Variable } & \multicolumn{3}{|c|}{ 15D tertiles (\%) } & \multirow[t]{2}{*}{ p-value* } \\
\hline & Lowest & Middle & Highest & \\
\hline Cardiovascular disease & 23.3 & 10.5 & 2.2 & $<0.001^{\dagger}$ \\
\hline Coronary artery disease & 20.8 & 8.3 & 1.6 & $<0.001^{+}$ \\
\hline Cerebrovascular disease & 7.0 & 2.6 & 0.6 & $<0.001^{+}$ \\
\hline Peripheral vascular disease & 3.8 & 1.3 & 0.0 & $0.001^{+}$ \\
\hline
\end{tabular}

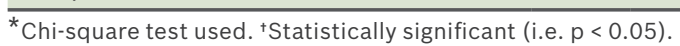

patients were married and employed. The mean duration of known T2DM was $10.1 \pm 8.1$ years. A majority $(60.7 \%)$ of the patients reported to be on oral agents alone for their DM, while $31.3 \%$ were on combination therapy (i.e. both oral agents and insulin) and $8.0 \%$ on insulin monotherapy. A majority of the patients suffered from at least one other (i.e. nondiabetic) chronic medical condition, such as dyslipidaemia (89.1\%) and hypertension $(80.2 \%)$. Most of the patients reported to be highly active (54.3\%). Patients without CVD were more likely to report a higher physical activity level (65.5\%) than those with CVD (34.5\%). Patients with CVD were found to consume higher total calories per day $(p=0.001)$. The majority of the patients did not smoke $(69.6 \%)$ and did not consume alcohol (89.5\%). A significantly higher percentage of patients with CVD, however, were smokers $(p=0.004)$ and alcohol drinkers $(p=0.025)$. In terms of biochemical and clinical characteristics, no significant differences were noted between the patients with CVD and those without, with respect to glycaemic control level, blood pressure, total cholesterol, and low-density lipoprotein cholesterol. There were, however, significant differences between the two groups in terms of WHR $(p=0.002)$, and high-density lipoprotein cholesterol $(p<0.001)$ and triglycerides $(p<0.013)$ levels.

Fig. 1 shows the 15D profile of the patients according to their CVD status. The mean 15D total score was significantly lower in the patients with CVD than in those without CVD $(p<0.001)$ (Table III). The patients with CVD were significantly worse off than those without CVD in all of the 15 dimensions: mobility $(p<0.001)$, vision $(p<0.001)$, hearing $(p=0.049)$, breathing $(p<0.001)$, sleeping $(p<0.001)$, eating $(p<0.001)$, speech ( $p=0.021)$, elimination $(p<0.001)$, usual activities ( $p<0.001)$, mental function ( $p<0.001)$, discomfort and symptoms $(p<0.001)$, depression $(p<0.001)$, distress $(p<0.01)$, vitality $(p<0.001)$, and sexual activity $(p<0.001)$.

The prevalences of CVD and its components, according to the 15D tertile groups, are shown in Table IV. CVD prevalence was significantly lower in the highest $15 \mathrm{D}$ tertile $(2.2 \%)$ when compared to the lowest and the middle $15 \mathrm{D}$ tertiles 
Table V. Statistically significant associations in the multinomial backward stepwise logistic regression analysis. Odds ratio for low HRQoL was measured using the 15D instrument.

\begin{tabular}{|c|c|c|c|c|c|c|}
\hline Variable & B & SE & Wald & df & OR $(95 \% \mathrm{Cl})$ & p-value \\
\hline Age (yrs) & 0.091 & 0.034 & 4.504 & 1 & $1.095(1.054-1.137)$ & $<0.001$ \\
\hline Duration of T2DM (yrs) & 0.081 & 0.025 & 10.404 & 1 & $1.085(1.032-1.140)$ & 0.001 \\
\hline BMI $\left(\mathrm{kg} / \mathrm{m}^{2}\right)$ & 0.072 & 0.034 & 4.504 & 1 & $1.074(1.006-1.148)$ & 0.034 \\
\hline $\begin{array}{l}\text { Ethnicity } \\
\text { Malay } \\
\text { Indian }\end{array}$ & -0.890 & 0.402 & 4.904 & 1 & $\begin{array}{c}0.411(0.187-0.903) \\
1\end{array}$ & 0.027 \\
\hline $\begin{array}{l}\text { PA } \\
\text { Low } \\
\text { High }\end{array}$ & 1.255 & 0.463 & 7.341 & 1 & $\begin{array}{c}3.506(1.415-8.689) \\
1\end{array}$ & 0.007 \\
\hline $\begin{array}{l}\text { CVD } \\
\text { Absent } \\
\text { Present }\end{array}$ & 2.463 & 0.445 & 30.471 & 1 & $\begin{array}{c}1 \\
11.746(4.898-28.167)\end{array}$ & $<0.001$ \\
\hline
\end{tabular}

The following variables were entered into the model: (a) continuous variables - age, duration of T2DM, BMI, and WHR; (b) categorical variables - gender (men vs women), ethnicity (Malay vs Chinese or Indian), marital status (married vs single/widowed/divorced), education level (college/university vs secondary school, primary school or no former education), smoking status (current smoker vs former smoker or nonsmoker), alcohol consumption (drinker vs former drinker or nondrinker), and physical activity (low PA vs moderate or high PA), CVD status (absent vs present).

$\mathrm{Cl}$ : confidence interval; CVD: cardiovascular disease; df: degrees of freedom; OR: odds ratio; PA: physical activity; SE: standard error; T2DM: type 2 diabetes mellitus

(23.3\% and $10.5 \%$, respectively) $(p<0.001)$. Similarly, the prevalence of CAD increased from $1.6 \%$ in the highest $15 \mathrm{D}$ tertile to $20.8 \%$ in the lowest $15 \mathrm{D}$ tertile $(\mathrm{p}<0.001)$. Similar trends were observed for cerebrovascular disease and PVD.

In the multinomial logistic regression analysis using backward stepwise method, CVD was found to be significantly associated with impaired HRQoL (OR 11.746; $\mathrm{p}<0.001$ ) (Table V). Impaired HRQoL was also significantly more common in patients who were older (OR 1.095; $\mathrm{p}<0.001)$ and in patients who had a longer duration of T2DM (OR 1.085; $p=0.001)$, a higher BMI (OR 1.047; $p=0.034)$, and a lower level of physical activity (OR 3.506; $p=0.007$ ). Malay patients were found to exhibit a lower likelihood of impaired HRQoL than Indian patients (OR 0.411; $\mathrm{p}=0.027$ ). No significant association was found between HRQOL and WHR, gender, marital status, education level, smoking status or alcohol consumption.

\section{DISCUSSION}

HRQoL is defined as an individual's perception of his/her symptoms, well-being, and physical and mental functional capacity; it is conceptually based on the World Health Organization's definition of health. ${ }^{(24)}$ The concept of HRQoL is wide-ranging, with different fields of human life influencing it. Its multidimensional nature and complexity present as a challenge for researchers. DM has a marked influence on HRQoL. The symptoms and accompanying complications of DM, together with its therapy, affect various aspects of an individual's HRQoL. ${ }^{(25)}$ Since CVD is the most prevalent complication in T2DM, the present study looked into the influence CVD has on the HRQoL of T2DM patients.

The findings of the present study confirmed that CVD was significantly associated with impaired HRQoL. T2DM patients with CVD had poorer HRQoL than those without CVD, with respect to all the dimensions of the 15D instrument. Impaired HRQoL was also significantly associated with advanced age and increased duration of T2DM. In our study, we found that patients of Malay ethnicity had significantly better HRQoL than patients of Indian ethnicity. Apart from that, higher BMI and lower physical activity negatively affected HRQoL. The lack of burdens significantly associated with T2DM alone (i.e. among the T2DM patients without CVD), on the aforementioned dimensions, was unexpected. It is possible that by the definitions of the present study, the T2DM patients without CVD did not have severe or advanced DM complications, or had T2DM for a relatively shorter duration, and would therefore have less HRQoL deficits detected on the 15D instrument. Earlier studies attributed impaired HRQoL in T2DM patients to the severity of DM and the manifestation of diabetic CVD. ${ }^{(26,27)}$ It is worth noting that the findings of the present study show that CVD is the strongest predictor of impaired HRQoL. This is in accordance with the findings of studies by Stafford et al and Li et al. ${ }^{(28,29)}$ In the Stafford et al study, patients with a stroke, heart attack or angina diagnosis were found to have the greatest decreases in EQ-5D HRQoL index scores. ${ }^{(28)}$ The study by Li et al highlighted that individuals with CVD had an approximately two- to four-fold increased likelihood of impaired HRQoL than individuals without CVD. ${ }^{(29)}$

Advanced age and a longer duration of T2DM were also found to have a detrimental impact on the HRQoL of our study cohort. This is in agreement with other reports. ${ }^{(2,30-34)} \mathrm{A}$ greater discriminative HRQoL score among older patients and patients with a longer duration of T2DM may be linked to these patients' reduced physical functioning, increased presence of comorbidities and reduced tolerance for the ambiguities associated with the disease. The results of the present study generally correspond well to the findings of previous research that show that the predilection of already-high-risk patients toward physical inactivity may hasten the burden of the disease and contribute toward altered functional outcomes of HRQoL, as evident from the predictive contribution of low physical activity level toward reduced HRQoL. ${ }^{(35)}$ 
The association between obesity and HRQoL has been documented in several studies. ${ }^{(36,37)}$ This association is consistent with our finding that elevated BMI, an indicator of obesity, is closely related to impaired HRQoL. Furthermore, in an American review of the effect of obesity on HRQoL in T2DM, the authors reported that decreased HRQoL was observed with increased body weight in all the studies reviewed. ${ }^{(38)}$ It is not surprising that cardiorespiratory performance and physical functioning may be impaired by obesity. This is because obese patients need to carry more weight while performing activities, and their larger physical size may make performing certain activities difficult. They may also experience low maximal oxygen uptake and psychosocial stress. All of this justifies the adverse effects of obesity on HRQoL.

In the present multiethnic hospital-based study, we also found that Indian T2DM patients experienced lower HRQoL than Malay T2DM patients. This concurs with the findings of an earlier study by Azman et al, which found that Malaysian Indians have lower HRQoL status in terms of physical functioning, bodily pain, energy fatigue/vitality, social functioning and mental health. ${ }^{(39)}$

If patients scored poorly in the breathing dimension of the $15 \mathrm{D}$ instrument, these patients probably suffered from coronary ischaemia and/or heart failure. The ischaemia may be asymptomatic or present with pain. A plausible explanation for painless infarction and ischaemic episodes in DM patients is autonomic neuropathy with involvement of the sensory supply to the heart. ${ }^{(40,41)}$ Meanwhile, a significant decrease in the mobility, usual activities and sexual activity dimensions may be due to the presence of angina pectoris, which could result in deterioration of physical capacity and effort tolerance, negatively affecting the HRQoL of a patient with CVD. In our study, patients with CVD also scored poorly in the discomfort and symptoms, and vitality dimensions when compared to patients without CVD. This indicates that patients experiencing threatening illnesses such as CAD, stroke or PVD have a worsened zest for life. It is also possible that lower vitality and decreased sexual activity may both be due to the adverse reactions of drugs, such as beta blockers and statins, which were commonly taken by patients with CVD in our cohort who were on more aggressive treatments.

In our study, T2DM patients with CVD also had poor 15D scores documented in the hearing, vision and mental function dimensions. Deterioration in hearing is multifactorial, and may be explained by advanced age and/or adverse reactions from medication use (e.g. frusemide). Ageing may also be a contributing factor to poor vision, along with glaucoma and diabetic retinopathy. Poor scores in mental function was not unexpected, as cognitive decline is often seen in elderly patients. Moreover, patients with CVD may experience silent ischaemia to brain tissues and speech difficulties secondary to stroke.

It is difficult to explain the mechanisms behind impaired sleeping among T2DM patients with CVD. The possible mediating mechanisms are most likely multifactorial. These mechanisms may be related to medications that can cause nocturia (e.g. diuretics, beta blockers or statins), as well as obesity, which is often associated with obstructive sleep apnoea and other sleep disturbances. We have no explanation as to why the results of the present study indicate a lower HRQoL in the eating and elimination dimensions among T2DM patients with CVD; the reasons remain unclear and are likely to be multifactorial. Finally, two dimensions of the 15D instrument that are of particular interest in our category of T2DM patients with CVD are mood disturbances such as depression and distress. These factors are often related to the patient's ability to cope with changes and losses. Consistent with the existing literature, ${ }^{(42,43)}$ we found that depression and distress were higher among the T2DM patients with CVD than those without CVD. This may primarily be due to daily medication requirements, dietary restrictions, active healthcare resource utilisation, and a sense of helplessness in dealing with the diseases. $^{(44)}$

The most noteworthy finding of the present study is the presence of significant predictions and associations between the HRQoL of T2DM patients and the following variables: CVD, age, duration of T2DM, ethnicity, physical activity level, and BMI. While other studies have reported extensively on the impact of CAD, stroke and PVD on HRQoL, the present study is one of the very few comprehensive studies evaluating the HRQoL of T2DM patients with and without CVD. The present study therefore makes a valuable contribution to the scientific literature on this topic. Our findings further underscore the importance of preventing and treating T2DM complications to prevent further deterioration in the HRQoL of T2DM patients. This study also highlights the need to identify factors that may be modulated to improve the HRQoL of T2DM patients.

The present study is limited by its cross-sectional nature. Although the assumption that CVD and its disorders precede declines in HRQoL has been supported by longitudinal studies, ${ }^{(28)}$ the temporal relationships could not be tested in the present study. Further prospective studies should be carried out to assess these temporal relationships. Another limitation is that only one measure of HRQoL (i.e. the 15D instrument) was used in the present study to test for comparability. While the $15 \mathrm{D}$ instrument has been extensively used in international studies, it is possible that alternative measures of HRQoL may accentuate different health conditions. It is important to keep in mind that the $15 \mathrm{D}$ instrument is not DM-specific, and it may thus reflect problems related to other conditions.

In conclusion, the presence of CVD was found to be significantly associated with a lower HRQoL in T2DM patients. Tertiary prevention is therefore of great importance in the effort to minimise the potential deterioration of HRQoL of T2DM patients, especially if the patient is also afflicted with CVD. Patients should also be empowered with adequate knowledge 
to enable the cultivation of better self-management skills, so as to further enhance their HRQoL.

\section{REFERENCES}

1. International Diabetes Federation. IDF Diabetes Atlas. 5th ed. Brussels, Belgium: IDF, 2011.

2. Hahl J, Hämäläinen $\mathrm{H}$, Sintonen $\mathrm{H}$, et al. Health-related quality of life in type 1 diabetes without or with symptoms of long-term complications. Qual Life Res 2002; 11:427-36.

3. Haffner SM, Letho S, Rönnemaa T, Pyörälä K, Laakso M. Mortality from coronary heart disease in subject with type 2 diabetes and in non diabetic subjects with and without prior myocardial infarction. N Engl J Med 1998; 339:229-34.

4. Diabetes Drafting Group. Prevalence of small vessel and large vessel disease in diabetic patients from 14 centres: The World Health Organization Multinational Study of Vascular Disease in Diabetics. Diabetologia 1985; 28:615-40

5. Assmann G, Schulte H. The Prospective Cardiovascular Münster (PROCAM) study: Prevalence of hyperlipidemia in persons with hypertension and/or diabetes mellitus and the relationship to coronary heart disease. Am Heart J 1988; 116:1713-24.

6. Stamler J, Vaccaro O, Neaton JD, Wentworth D. Diabetes, other risk factors, and 12-yr cardiovascular mortality for men screened in the Multiple Risk Factor Intervention Trial. Diabetes Care 1993;16:434-44.

7. Gu K, Cowie CC, Harris MI. Diabetes and decline in heart disease mortality in US adults. JAMA 1999; 281:1291-7.

8. Fox CS. Cardiovascular disease risk factors, type 2 diabetes mellitus, and the Framingham Heart Study. Trends Cardiovas Med 2010; 20:90-5.

9. Fisher M, McMurray JJ. Diabetic cardiology. London: John Wiley \& Sons, 2007.

10. American Heart Association. Heart Disease and Stroke Statistics: 2009 Update. Dallas: American Heart Association, 2009.

11. Stern MP. Glycemia and cardiovascular risk. Diabetes Care 1997; 20:1501-2.

12. Laakso M. Hyperglycemia and cardiovascular disease in type 2 diabetes. Diabetes 1999; 48:937-42.

13. Morrish NJ, Wang SL, Stevens LK, Fuller JH, Keen K. Mortality and causes of death in the WHO multinational study of vascular disease in diabetes. Diabetologia 2001; 44 Suppl 2:S14-21.

14. Lloyd A, Sawyer W, Hopkinson P. Impact of long-term complications on quality of life in patients with type 2 diabetes not using insulin. Value Health 2001; 4:392-400.

15. The Criteria Committee of the New York Heart Association. Nomenclature and criteria of diseases of the heart and blood vessels. Boston: Little, Brown and company, 1973.

16. Kaplan RM. Health-related quality of life in cardiovascular disease. J Consult Clin Psychol 1988; 56:382-92.

17. Sintonen $\mathrm{H}$. The $15 \mathrm{D}$ instrument of health-related quality of life: Properties and applications. Ann Med 2001; 33:328-36.

18. Lee RD, Nieman DC. Nutritional Assessment. 5th ed. New York: McGrawHill Higher Education, 2010.

19. International Physical Activity Questionnaire Research Committee. Guidelines for Data Processing and Analysis of the International Physical Activity Questionnaire (IPAQ). November 2005. Available at: www.ipaq. ki.se. Accessed August 1, 2011.

20. World Health Organization. Health topics. Reproductive health. Available at: www.who.int/topics/reproductive_health/en. Accessed September 1, 2011.

21. Sintonen $\mathrm{H}$. Outcome measurements in acid-related diseases. Pharmacoeconomics 1994; 5:17-26.

22. Sintonen $\mathrm{H}$. The 15D-measure of health-related quality of life. I. Reliability, validity and sensitivity of its health state descriptive system. Working Paper 41. Melbourne: National Centre for Health Program Evaluation, 1994.
23. Sintonen H. The 15D-measure of health-related quality of life. II. Feasibility, reliability and validity of its valuation system. Working Paper 42. Melbourne: National Centre for Health Program Evaluation, 1995.

24. Testa MA, Simonson DC. Assessment of quality-of-life outcomes. N Engl J Med 1996; 334:835-40.

25. Rubin RR, Peyrot M. Quality of life and diabetes. Diabetes Metab Res Rev 1999; 15:205-18.

26. Wändell PE, Brorsson B, Åberg H. Functioning and well-being of patients with type 2 diabetes or angina pectoris, compared with the general population. Diabetes Metab 2000; 26:465-71.

27. Unsar S, Sut N, Durna Z. Health-related quality of life in patients with coronary artery disease. J Cardiovasc Nurs 2007; 22:501-7.

28. Stafford M, Soljak M, Pledge V, Mindell J. Socio-economic differences in the health-related quality of life impact of cardiovascular conditions. Eur J Public Health 2012; 22:301-5.

29. Li C, Ford ES, Mokdad AH, et al. Clustering of cardiovascular disease risk factors and health-related quality of life among US adults. Value Health 2008; 11:689-99.

30. Ahola AJ, Saraheimo M, Forsblom C, et al. Health-related quality of life in patients with type 1 diabetes-association with diabetic complications (The FinDiane Study). Nephrol Dial Transplant 2010; 25:1903-8.

31. Akinci F1, Yildirim A, Gözü $H$, et al. Assessment of health-related quality of life (HRQoL) of patients with type 2 diabetes in Turkey. Diabetes Res Clin Pract 2008; 79:117-23.

32. Hänninen J, Takala J, Keinänen-Kiukaanniemi S. Quality of life in NIDDM patients assessed with the SF-20 questionnaire. Diabetes Res Clin Pract 1998; 42:17-27.

33. Papadopoulos AA, Kontodimopoulos N, Frydas A, Ikonomakis E, Niakas D. Predictors of health-related quality of life in type II diabetic patients in Greece. BMC Public Health 2007; 7:186.

34. Järvinena $O$, Saarinen $T$, Julkunen J, Huhtala $H$, Tarkka MR. Changes in health-related quality of life and functional capacity following coronary artery bypass graft surgery. Eur J Cardiothorac Surg 2003; 24:750-6.

35. Fischbacher CM, Hunt S, Alexander L. How physically active are South Asians in the United Kingdom? A literature review. J Public Health (Oxf) 2004; 26:250-8.

36. Miettola J, Niskanen LK, Viinamaki H, Sintonen H, Kumpusalo E. Metabolic syndrome is associated with impaired health-related quality of life: Lapinlahti 2005 study. Qual Life Res 2008; 17:1055-62.

37. Hlatky MA, Chung S-C, Escobedo J, et al. The effect of obesity on quality of life in patients with diabetes and coronary artery disease. Am Heart J 2010; 159:292-300.

38. Dennett SL, Boye KS, Yurgin NR. The impact of body weight on patient utilities with or without type 2 diabetes: A review of the medical literature. Value Health 2008; 11:478-86.

39. Azman AB, Sararaks S, Rugayah B, et al. Quality of life of the Malaysian General Population: Results from a postal survey using the SF-36. Med J Malaysia 2003; 58:694-711.

40. Johnstone MT, Kinzfogl GP. Contemporary Cardiology: Diabetes and Cardiovascular Disease. 2nd ed. New Jersy: Humana Press Inc, 2005.

41. Shaw JE, Punjabi NM, Wilding JP, Alberti KG, Zimmet PZ. Sleep-disordered breathing and type 2 diabetes: A report from the International Diabetes Federation Taskforce on Epidemiology and Prevention. Diabetes Res Clin Pract 2008; 81:2-12.

42. Durmaz T, Özdemir Ö, Özdemir BA, et al. Factors affecting quality of life in patients with coronary heart disease. Turk J Med Sci 2009; 39:343-51.

43. Liles DR, Kallen MA, Petersen LA, Bush RL. Quality of life and peripheral arterial disease. J Surg Res 2006; 136:294-301.

44. Anderson RM, Fitzgerald JT, Wisdom K, Davis WK, Hiss RG. A comparison of global versus disease- specific quality-of-life measures in patients with NIDDM. Diabetes Care 1997; 20:299-305. 